\title{
Effect of convective outer layers modeling on non-adiabatic seismic observables of $\delta$ Scuti stars
}

\author{
J. Montalbán ${ }^{1}$ and M.-A. Dupret ${ }^{2}$ \\ 1 Institut d'Astrophysique et de Géophysique de l'Université de Liège, Belgium \\ e-mail: j.montalban@ulg.ac.be \\ 2 Observatoire de Paris, LESIA, 92195 Meudon, France
}

Received 6 November 2006 / Accepted 13 March 2007

\begin{abstract}
Context. The identification of pulsation modes in $\delta$ Scuti stars is mandatory for constraining the theoretical stellar models. The nonadiabatic observables used in the photometric identification methods depend, however, on convection modeling in the external layers. Aims. We determine how the treatment of convection in the atmospheric and sub-atmospheric layers affects the mode identification and what information about the thermal structure of the external layers can be obtained from amplitude ratios and phase lags in Strömgren photometric bands.

Methods. We derive non-adiabatic parameters for $\delta$ Scuti stars by using, for the first time, stellar models with the same treatment of convection in the interior and in the atmosphere. We compute classical non-gray mixing length models, and as well non-gray "full spectrum of turbulence" (FST) models. Furthermore, we compute the photometric amplitudes and phases of pulsation by using the colors and the limb-darkening coefficients as derived from the same atmosphere models as used in the stellar modeling.

Results. We show that the non-adiabatic phase-lag is mainly sensitive to the thermal gradients in the external layers, hence to the treatment of convection, and that this sensitivity is also clearly reflected in the multi-color photometric phase differences.
\end{abstract}

Key words. convection - stars: atmospheres - stars: oscillations - stars: variables: $\delta$ Scuti - stars: evolution

\section{Introduction}

Asteroseismology uses the frequency of stellar pulsations as a probe of the interior stellar structure, by comparing the observed frequencies with those determined from stellar models. In order to constrain the physical parameters of a star from its pulsation frequencies, it is necessary to identify the modes of pulsation, that is, to determine the spherical harmonic degree $\ell$, the azimuthal order $m$, and the radial order $n$ for each mode. This is far from trivial in $\delta$ Scuti stars, as their excited modes do not fall in the asymptotic range and, furthermore, rotation and possible coupling destroy any pattern regularity. As a consequence, mode identification is difficult and other complementary observables must be used for this purpose.

The mode identification photometric methods are based on the analysis of luminosity variations in different photometric passbands and on the dependence of their amplitudes and phases on the spherical harmonic degree, $\ell$. These techniques are based on the theoretical study by Dziembowski (1977) of the light variations generated by non-radial oscillation. Afterwards, several improvements were made by Balona \& Stobie (1979), Stamford \& Watson (1981), Watson (1988), Garrido et al. (1990), and Heynderickx et al. (1994). The main shortcoming of these approaches is that the non-adiabatic amplitude and phase of the effective temperature are treated as free parameters. Cugier et al. (1994) updated the method by using Dziembowski's nonadiabatic code to derive amplitude and phases and they applied it to $\beta$ Cep stars. This last approach does not take the non-adiabatic eigenfunctions in the atmosphere layers into account and assumes the Lagrangian temperature variation to be equal to the local effective temperature variation at the photosphere. Dupret et al. (2002) show, however, that the temperature perturbation in the photosphere is rather different from the local effective temperature variation, because of the significant optical depth variation produced by the oscillation.

Since the non-adiabatic observables (and hence multi-color magnitude variation) strongly depend on the variation in the atmospheric thermal structure, their accurate determination requires solving the adequate non-adiabatic equations in the stellar atmosphere as well. Dupret et al. (2003) include an improved treatment of the oscillations in the non-gray atmosphere. The application of their model to $\beta$ Cephei and SPB stars (Dupret et al. 2003) offers an improvement in mode identification.

Stars in the Hertzprung-Russell Diagram (HRD) region where $\delta$ Scuti stars are located should have, according to theoretical stellar models, an external structure with one or two convective regions due to $\mathrm{H}$ and $\mathrm{He}$ ionization. Since these regions are very shallow, they are also very over-adiabatic. As a consequence, the thermal structure of the outer layers, and therefore the photometric amplitude ratios and phases differences in different bands, are very sensitive to the details of convection modeling, such as Balona \& Evers (1999) show in the first nonadiabatic study of $\delta$ Scuti stars using the Dziembowski's code.

The "standard model" of convection in stellar evolution is the mixing-length theory (MLT Böhm-Vitense 1958), where turbulence is described by a relatively simple model. This essentially contains one adjustable parameter, $\alpha$, relating the mixing length to the local pressure scale height. Several recent papers have discussed the effect on the non-adiabatic observables of varying $\alpha$ (Daszyńska-Daszkiewicz et al. 2003; Moya et al. 2004; Daszyńska-Daszkiewicz et al. 2005). However, in those papers, the convection treatment is changed only in the regions computed with the corresponding stellar structure code, and not in 
the atmospheric layers ${ }^{1}$, where the multi-color magnitude variations due to oscillations are generated.

The analysis of the effect of convection modeling on stellar structure and evolution is not always obvious, since the available atmosphere models are typically computed for a given theory of convection and for a fixed value of $\alpha$-MLT. Luckily, Heiter et al. (2002) have published new atmosphere model grids based on Kurucz's ATLAS9 code. In this new grid (thereafter NEMO grid), the atmosphere models were computed for different treatments of convection: MLT (with two different values of the $\alpha$ parameter), Canuto \& Mazzitelli (1991a) (CM), and Canuto et al. (1996) (CGM) treatments (also known as FST - full spectrum of turbulence-treatments). These choices are extensively explained in Sect. 2 of Heiter et al. (2002), and the main characteristics and differences among these convection treatments will be described in Sect. 2.

By including the NEMO grids in our model computations, we are able to self-consistently study the effect of convection treatment on the oscillation amplitudes and phases in different passbands. The analysis presented here is consistent on two levels: first, the stellar models (interior and atmosphere) used in the non-adiabatic computations were obtained by using the same (either FST or MLT) convection treatment; and second, the color-transformation tables, as well as the limb-darkening law derivation, are based on the same atmosphere models as those we included in the stellar structure modeling. In Sect. 2, we present the models used in this study: the interior equilibrium models (Sect. 2.1), the atmosphere models and limb-darkening (Sect. 2.3), the non-adiabatic oscillation code (Sect. 2.4), and the color transformations giving the observable properties of $\delta$ Scuti pulsations (Sect. 2.5). In Sect. 2.2, we provide an outline of the problem of modeling convection in A-type stars.

One of the main results of Heiter et al. (2002) is that MLT models with a small mixing-length parameter (e.g., $\alpha \sim 0.5)$ are equivalent, in the atmospheric region where the observed flux originates, to FST models. Both treatments indeed predict a low convective efficiency for these layers. The thermal structure in the subphotospheric layers for models with MLT $(\alpha=0.5)$ and FST may be, however, quite different. We study whether the nonadiabatic features of $\delta$-Scuti oscillations are sensitive to these differences.

In Sect. 3 we compare the results obtained with MLT and FST treatments and explain the origin of the differences. We analyze as well the respective weights of the over-adiabatic structure and of the color transformations on the theoretical amplitude ratios and on phase differences in Strömgren photometric passbands. Finally, we consider the application to a particular $\delta$ Scuti star in Sect. 4, AN Lyn, and, in Sect. 5, we present our conclusions.

\section{Theoretical models}

\subsection{Stellar models}

The stellar models were computed by means of the stellar evolution code ATON3.0, recently updated to be used in asteroseismology modeling (D'Antona et al. 2005). We computed models for a metal mass fraction $Z=0.02$ and assumed a helium mass fraction $Y=0.28$. At temperatures $T \geq 12000 \mathrm{~K}$, we adopted the OPAL radiative opacities $(\bar{\kappa})$ (Iglesias \& Rogers 1996) for the solar Z-distribution from Grevesse \& Noels (1993). In the

1 The exact optical depth, $\tau=2 / 3$ or $\tau=10$ depends on the particular stellar evolution code used. high-density $(\rho)$ regions, the opacities are linearly extrapolated $(\log \bar{\kappa}$ vs. $\log \rho$ ) and harmonically added to conductive opacities by Itoh \& Kohyama (1993). At lower temperatures we used Alexander \& Ferguson (1994) molecular opacities (plus electron conduction in full ionization) for the same $\mathrm{H} / \mathrm{He}$ ratios as in the OPAL case. A complete description of the equation of state (EOS) used in ATON3.0 code is given in D'Antona et al. (2005). In short, this code uses OPAL(2001) EoS (Rogers \& Nayfonov 2002) and Saumon et al. (1995) EoS for the low-temperature and high-density domain.

Several treatments of convection are available in ATON3.0: the classic mixing length theory (MLT) model, with the Cox \& Giuli (1968) formulation, and two different FST models, according to Canuto \& Mazzitelli (1991b) and Canuto et al. (1996) approaches. A detailed description of the convection modeling in ATON3.0 is provided by Ventura et al. (1998). For the present study, we adopted MLT and Canuto et al. (1996, thereafter FST or CGM) models. Except for explicitly mentioned cases, the $\alpha$-MLT and the $\alpha^{*}$-FST parameters were the same as those in the atmosphere models.

As surface boundary conditions (for detail, see Montalbán et al. 2004) we took the NEMO atmosphere grids. As mentioned above, these new atmosphere models were computed using MLT model (with $\alpha=0.5$ and, for a smaller range of parameters, $\alpha=1.25$ as in the classic ATLAS9 model) and also CGM with $\alpha^{*}=0.09$ (Canuto et al. 1996). That allowed us to consistently build stellar models (interiors and atmospheres). The boundary was located at optical depth $\tau_{\mathrm{ph}}=10$, as suggested by Heiter et al. (2002), except when MLT models were computed with a $\alpha$-MLT value in the interior different from that of the atmosphere. In that case, the boundary was located at $\tau_{\mathrm{ph}}=1$, to avoid mixing of different convection efficiencies in the overadiabatic region.

Helium and metal gravitational settling were not included in these computations. All the models were computed following the evolution from the pre-main sequence (PMS) phase. As numerical resolution is concerned, the number of models for the PMS phase was on the order of 600, and the evolution from the ZAMS to the TAMS was done in 350 models; besides, 2500-3000 mesh points were used to describe the stellar structure.

Before applying non-adiabatic computations, the stellar structure of the equilibrium model was extended by matching the atmosphere structure (from $\tau_{\mathrm{ph}}$ up to $\tau=10^{-4}$ ), as obtained by interpolation in the grid of atmosphere models.

\subsection{Convection modeling in A-type stars}

Standard models for A-type stars predict stellar structures with a convective core and one or two external convective regions, the inner one corresponding to HeII ionization, and the shallower and outer one to that of $\mathrm{HI}$ and HeI. Convection in the central region is almost adiabatic, while the external convective regions are highly overadiabatic, but very thin and shallow. As a consequence, the details of convection in A-type stars (except for the overshooting that changes the dimension of the convective core) have a negligible effect on their location in the HR diagram.

Nevertheless, the convective heat transport changes the temperature gradient and affects, therefore, the spectral line profiles and the emission in different photometric bands. In fact, there is spectroscopic evidence of the presence of convection in A-type stars, such as the peculiar behavior of Balmer lines as a function of temperature, the shape of bisectors, or chromospheric activity (see Smalley 2004, for a review). These observations 
suggest that convective velocity fields of several $\mathrm{km} \mathrm{s}^{-1}$ are present in A-stars, while the temperature gradient must be close to the radiative one, except for the coolest objects $(\sim 7000 \mathrm{~K})$.

It has been already shown by Fuhrmann et al. (1993, 1994), van't Veer-Menneret \& Megessier (1996), van't Veer-Menneret et al. (1998), Barklem et al. (2002), Smalley \& Kupka (1997, 1998), and Smalley et al. (2002), that spectroscopic and photometric features seem better-fitted by adopting low-efficiency convection models (for instance, MLT with $\alpha=0.5$ or CGM) that provide temperature gradient close to the radiative one. Nevertheless, neither MLT nor CGM succeed in matching all the photometric features in the A-F stars' effective temperature domain.

Other observational evidence in favor of low-efficient convection in A-type stars has been provided by DaszyńskaDaszkiewicz et al. (2003) and Daszyńska-Daszkiewicz et al. (2005) in their fits of the seismic features of several $\delta$ Scuti stars.

Both non-local models of convection (Kupka \& Montgomery 2002) and 2D and 3D numerical simulations (Ludwig et al. 1999; Freytag \& Steffen 2004) indicate that, in order to reproduce the convective flux in the external layers, the value of the MLT- $\alpha$ parameter should vary from 0.5 at $8000 \mathrm{~K}$ to $\sim 1$ or higher at $7000 \mathrm{~K}$. On the other hand, the $\alpha$ value required to reproduce the flux in the HeII convective zone should also be four times higher than what is used in the HI, HeI convective zone. Fortunately (or unfortunately) for what concerns the present study, Moya et al. (2004) have shown that a change of convection efficiency in the HeII convective region does not affect the excited modes of $\delta$ Scuti stars.

In the computation presented in this paper, we use the mixing length theory (Böhm-Vitense 1958) and the "full spectrum of turbulence" formalism (CGM). The main improvement in CGM with respect to MLT is that, while the latter treats the heat transport by mimicking the spectral distribution of eddies by one "average" eddy (reliable only for high viscosity fluids), the former overcomes the one-eddy approximation by using a turbulence model to compute the full spectrum of a turbulent convective flow. Canuto (1996) show that a consequence of this different heat transport is that, in the limit of highly efficient convection ( $S \gg 1$, where $S$ is the convective efficiency, see e.g. CM for details), MLT underestimates the convective flux, while - in the low-efficiency limit - MLT overestimates it. Thus, CGM convective fluxes are $\sim 10$ times larger than the MLT ones for the $S \gg 1$ limit, while the CGM flux is $\sim 0.3$ times the MLT one in the low-efficiency limit $(S \ll 1)$. This behavior yields steeper temperature gradients for FST than for solar-tuned MLT models in the over-adiabatic region at the top of a convection zone. In inefficient convection, the temperature gradient sticks to the radiative one and starts splitting only when convection becomes efficient. Another difference between CGM and MLT concerns the length scale $(\Lambda)$ for transport processes: $\Lambda_{\mathrm{MLT}}=\alpha \cdot H_{\mathrm{p}}$ and $\Lambda_{\mathrm{CGM}}=z+\alpha^{*} H_{\mathrm{p} \text {,top }}$, where $z$ is the distance to a boundary of convective region, and the second term is a fine-tuning parameter that allows small adjustments, if exact stellar radii are needed (e.g., in helioseismology). As Canuto et al. (1996) stress, the role of $\alpha^{*}$ in solar FST models is radically different from that of $\alpha$ in the MLT model. In fact, FST tuning affects only layers close to the boundaries. For inner layers, $z$ quickly grows and becomes

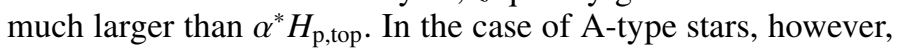
the small dimension of convective zones implies that this term could be a significant fraction of $z$. For the models presented in this paper, we use $\alpha^{*}=0.09$, as derived by Canuto et al. (1996) from solar calibration ${ }^{2}$ and adopted by Heiter et al. (2002) for their atmosphere models.

\subsection{Atmosphere models and limb darkening}

The original ATLAS 9 code treats convection by using MLT with $\alpha=1.25$ and has the possibility of including a sort of overshooting (see Castelli et al. 1997b,a, for details). The value $\alpha=1.25$ and the option of "approximate overshooting" were originally adopted to fit the intensity spectrum at the center of the solar disk and the solar irradiance. However, Castelli et al. (1997b) show that these quantities are much more sensitive to the overshooting-on mode than to the value of $\alpha$ itself (see also Heiter et al. 2002). The new grids of ATLAS9 atmospheres by Heiter et al. (2002), which we used in our computations, introduce some improvements with respect to the original ones published by Kurucz (1993) and Castelli et al. (1997b); in particular, they provide a finer grid spacing $\left(\Delta T_{\mathrm{eff}}, \Delta \log g\right)$, as well as a higher vertical resolution. For the reader's convenience, we recall the main characteristics of these grids:

i) Three different models of convection are considered: $\operatorname{MLT}(\alpha=0.5)$, FST according to CM and to CGM.

ii) For FST models, the vertical resolution in the atmospheric integration is increased from 72 to 288 layers, spanning the range from $\log \tau_{\text {Ross }}=-6.875$ to $\log \tau_{\text {Ross }}=2.094$ (where $\tau_{\text {Ross }}$ is the average Rosseland optical depth).

iii) Effective temperature range: from 4000 to $10000 \mathrm{~K}$, with $\Delta T_{\text {eff }}=200 \mathrm{~K}$.

iv) Gravity $(\log g$ ) from 2.0 to 5.0 , with $\Delta \log g=0.2$. In the new ATLAS9-MLT grids, the convection is described as in Castelli (1996) and Castelli et al. (1997b) (that is $V / A=\Lambda / 6$ and $y=0.5)$, but with $\alpha=0.5$ and without "overshooting".

In the CGM model atmospheres, the convective flux is computed as in Canuto et al. (1996), but the characteristic scale length is defined as $\Lambda=\min \left(z_{\text {top }}+\alpha^{*} H_{\mathrm{p} \text {,top }}, z_{\mathrm{bot}}+\alpha^{*} H_{\mathrm{p}, \text { bot }}\right)$ where the index "top" and "bot" refers to the top and bottom of the convective region, and $\alpha^{*}=0.09$ (see footnote 2).

The photometric methods (Sect. 2.5) used for $\delta$ Scuti mode identification are based on an analytical expression of the monochromatic oscillation amplitudes and phases. In this expression (Eq. (1)) the weighted limb-darkening integrals $\left(b_{\ell, \lambda}\right.$, Eq. (2)) explicitly appear along with their derivatives with respect to $\log T_{\text {eff }}$ and $\log g$ (Eqs. (3) and (4)). The new and denser grid of model atmospheres allows smoother $b_{\ell, \lambda}$ to be derived, with their derivatives as required for mode identification method (Garrido 2000).

In this paper we use the limb-darkening coefficients (LDC) for the Strömgren photometric system, as derived by Barban et al. (2003) by using the quadratic-law, for a sample of new atmosphere models with effective temperature between 6000 and $8500 \mathrm{~K}, \log g$ between 2.5 and 4.5 , and solar metallicity. These authors determine, for each $u v b y$ band, the best limbdarkening law, as well as the integrals $b_{\ell, \lambda}$ and find similar results for CGM and MLT $(\alpha=0.5)$, but significant differences between CGM and the usual MLT $(\alpha=1.25)$. Besides, they show that the differences in the LDCs follow those in the vertical temperature structure of the atmosphere model due to the different treatments of convective transport. The differences between CGM and $\operatorname{MLT}(\alpha=1.25)$ models are higher for low temperatures, but

\footnotetext{
2 Note that this value was derived for the solar calibration using grayatmosphere as external boundary conditions.
} 
the differences propagate toward high temperatures for model atmosphere with higher gravities. The differences between LDC's computed for MLT $(\alpha=0.5)$ and CGM atmosphere models are much smaller than for the $\operatorname{MLT}(\alpha=1.25)$, but they can reach the same order of magnitude as for $\operatorname{MLT}(\alpha=1.25)$ when intermediate gravity and temperature $\sim 7600 \mathrm{~K}$ are considered. As a general statement, the differences in the $b_{\ell}$ coefficients and derivatives computed with CGM and MLT $(\alpha=0.5)$ are much smaller $(\sim 5 \%)$ than differences obtained between computations with CGM and MLT $(\alpha=1.25)(\sim 20 \%)$.

As for the LDCs, the effect of convection treatment on $b_{\ell}$ is maximum at low effective temperature and low gravity models, and these large differences extend towards higher temperatures for models with high gravity. Furthermore, the magnitude of the differences in $b_{\ell}$ due to the convection treatment is $\ell$-dependent.

The use of a higher resolution in optical depth in the model atmospheres and the different treatment of the convection imply significant changes in the LDC values. In this paper we use these results to analyze the effect of LDCs and of the external thermal stratification on the theoretically determined amplitude ratios and phases.

\subsection{Non-adiabatic treatment}

We do a non-adiabatic seismic analysis of our models by using the code MAD (Dupret et al. 2003). In this code the adiabatic eigenfunctions are used as trial input for the non-adiabatic computations. The input model is a stellar model extended with the atmosphere corresponding to its $T_{\text {eff }}, \log g$ and adopted convection treatment. In this context, we use the Heiter et al. (2002) atmosphere models for the first time.

Since the convection-pulsation interaction implemented in MAD cannot be applied to the FST formalism, we adopt the frozen convection flux approximation, that is, we neglect the Lagrangian variation of the convective luminosity (radial component) and the Lagrangian variation of the transversal component of the convective flux. This approximation is not appropriate for describing the red edge of $\delta$ Scuti instability strip. Nevertheless, given the comparative character of this work and the low efficiency of convection for most of the models considered here ${ }^{3}$, we expect the convection-pulsation interaction not to be relevant to this study. The transfer equation treatment in the MAD code is different in the interior and in the pulsating atmosphere (Dupret et al. 2003). In the latter, the hypothesis at the base of the procedure is not justified for convective regions, so that the match layer between the interior and the atmosphere must be located outside the convective envelope. Even, if in the extended stellar model, the match between atmosphere and interior is made in a convective region, and the properties of a given layer come formally from the atmosphere model, the non-adiabatic treatment reserved to the atmosphere will be apply only to layers such that the convective to radiative luminosity ratio is lower than $10^{-10}$.

These computations make it possible to derive the phase lag $\left(\psi_{\mathrm{T}}\right)$ between the local relative variation of the effective temperature and the relative radial displacement $(\delta r)$ and the corresponding amplitude ratio $\left(f_{\mathrm{T}}\right)$, which are basic ingredients in determining the magnitude variations in different photometric passbands (see next section).

\footnotetext{
${ }^{3}$ FST models and MLT ones with $\alpha$ parameter smaller than one.
}

\subsection{Multi-color photometry}

The non-adiabatic quantities $\psi_{\mathrm{T}}=\phi\left(\delta T_{\mathrm{eff}} / T_{\mathrm{eff}}\right)-\phi(\delta r / R), f_{\mathrm{T}}=$ $\left|\delta T_{\text {eff }} / T_{\text {eff }}\right| /|\delta r / R|$ and $f_{\mathrm{g}}=\left|\delta g_{\text {eff }} / g_{\text {eff }}\right|$ (where $g_{\text {eff }}$ is the effective gravity and $\delta g$ the variation of gravity at the photosphere) can be related to photometric observables such as the amplitude ratios and phase differences between magnitude variations in different photometric passbands. It has, in fact, been shown (e.g. Watson 1988, and references therein) that, in a one-layer linear approximation, the magnitude variation at a wavelength $\lambda$ produced by a stellar pulsation with spherical harmonic degree $\ell$, azimuthal order $m$ and angular frequency $\sigma$ is given by

$$
\begin{aligned}
\delta m_{\lambda}= & -\frac{2.5}{\ln 10} a P_{\ell}^{m}(\cos i) b_{\ell \lambda}\{-(\ell-1)(\ell+2) \cos (\sigma t) \\
& +f_{\mathrm{T}} \cos \left(\sigma t+\Psi_{\mathrm{T}}\right)\left(\alpha_{\mathrm{T} \lambda}+\beta_{\mathrm{T} \lambda}\right) \\
& \left.-f_{\mathrm{g}} \cos (\sigma t)\left(\alpha_{\mathrm{g} \lambda}+\beta_{\mathrm{g} \lambda}\right)\right\}
\end{aligned}
$$

where $i$ is the inclination angle between the stellar rotation axis and the observer line of sight, $a$ is the amplitude of the relative radial displacement at the photosphere, and $P_{\ell}^{m}$ is the associated Legendre function of degree $\ell$ and azimuthal order $m$. Then, $b_{\ell \lambda}$ is related to the limb-darkening law by

$b_{\ell \lambda}=\int_{0}^{1} h_{\lambda} \mu P_{\ell} \mathrm{d} \mu$

where $h_{\lambda}$ is the normalized limb-darkening function, and $\mu=$ $\cos \theta$ with $\theta$ the angle between the line of sight and the normal to the local stellar surface. The rest of quantities appearing in Eq. (1): $\alpha_{\mathrm{T} \lambda}, \beta_{\mathrm{T} \lambda}, \alpha_{\mathrm{g} \lambda}$, and $\beta_{\mathrm{g} \lambda}$ are derived from the appropriate atmosphere model with effective temperature $T_{\text {eff }}$ gravity $\log g$ and given convection treatment:

$\alpha_{\mathrm{T} \lambda}=\frac{\partial \ln F_{\lambda}^{+}}{\partial \ln T_{\mathrm{eff}}} ; \quad \beta_{\mathrm{T} \lambda}=\frac{\partial \ln b_{\ell \lambda}}{\partial \ln T_{\mathrm{eff}}} ;$

$\alpha_{\mathrm{g} \lambda}=\frac{\partial \ln F_{\lambda}^{+}}{\partial \ln g_{\mathrm{eff}}} ; \quad \beta_{\mathrm{g} \lambda}=\frac{\partial \ln b_{\ell \lambda}}{\partial \ln g_{\mathrm{eff}}}$.

An appropriate way to test multi-color theoretical predictions is to construct phase-amplitude diagrams corresponding to wellchosen combinations of photometric bands (e.g. Garrido et al. 1990). The theoretical results corresponding to modes of different spherical degrees $\ell$ occupy very distinct regions. This makes the identification of $\ell$ possible by searching for the best fit between theory and observations. The non-adiabatic quantities $f_{\mathrm{T}}$ and $\psi_{\mathrm{T}}$, which play a major role in Eq. (1), are highly sensitive to the characteristic of the convective envelope (see Figs. 7 and 8). On the other hand, Barban et al. (2003) show that the sensitivity of $b_{\ell \lambda}$ and its derivatives to the convection model are different for different photometric passbands and different degree $\ell$. We expect, therefore, that constraints on the characteristics of the convective envelope could be obtained from very precise multicolor photometric observations.

\section{General results for $\delta$ Scuti stars}

As already mentioned, thanks to the shallow convection envelope in A-type, the HRD location of a stellar model of fixed mass and chemical composition is not affected by the treatment of non-adiabatic convection layers. This fact allows us to easily separate the effect of convection. We have computed models for 1.8 and $2.0 M_{\odot}$ with the FST formalism and with MLT $(\alpha=0.5)$. We have also computed MLT models with $\alpha_{\text {int }}=0.1$, 


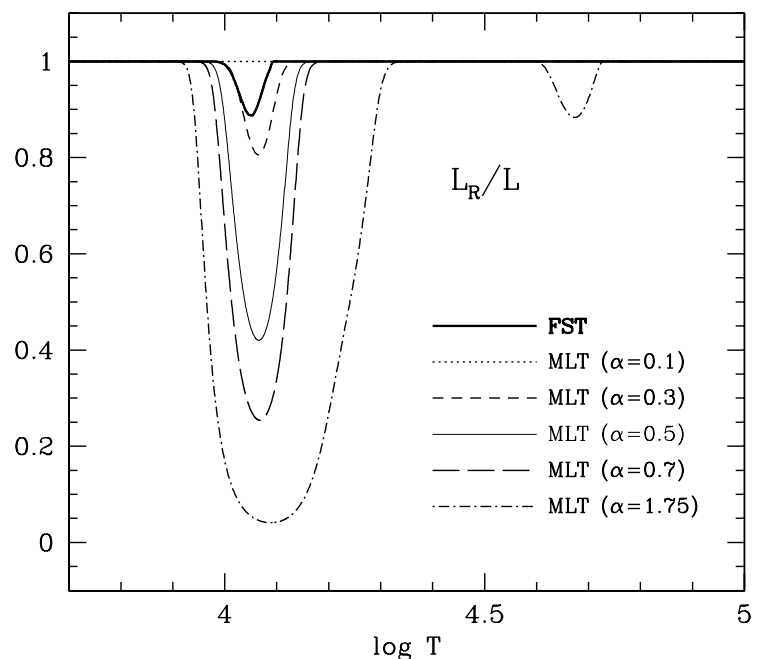

Fig. 1. Fraction of luminosity transported by radiation with respect to total luminosity as a function of temperature for several $1.8 M_{\odot}$ models in the same HRD location $\left(T_{\text {eff }}=7400 \mathrm{~K}, \log g=3.98\right)$ but computed with different treatments of convection. The FST model was computed with the FST convection in the interior and FST atmospheres by Heiter et al. (2002). The $\operatorname{MLT}(\alpha=0.5)$ model implies $\alpha=0.5$ in the interior and the corresponding $\alpha=0.5$ Heiter et al. (2002) atmosphere. The MLT models with $\alpha \neq 0.5$ were computed with the indicated $\alpha$ value in the interior up to the optical depth $\tau=1$ and $\operatorname{MLT}(\alpha=0.5)$ atmosphere model for $\tau<1$.

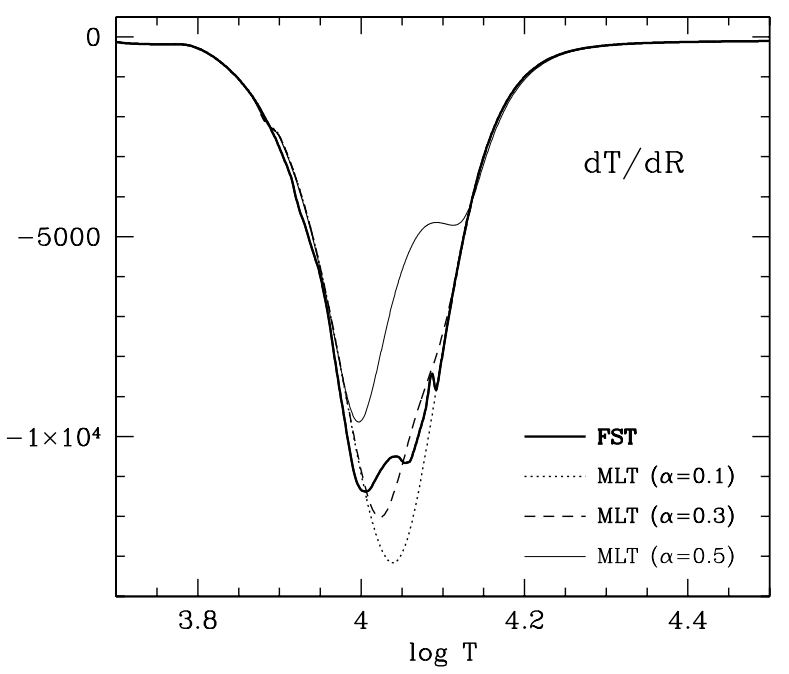

Fig. 2. Temperature gradient as a function of temperature inside the star, for some of the models in Fig. 1.

$0.3,0.7$, and $1.75^{4}$. Even if for a given central hydrogen content, all the computations yield the same point in the HRD, the characteristics of the external layers are affected by different convection treatments. The two main physical quantities affected by this treatment are the energy fraction transported by convection (Fig. 1) and the temperature gradient (Fig. 2). As is well known, the energy fraction transported by convection increases as

${ }^{4}$ We would like to call attention to the fact that since the available atmosphere models use $\operatorname{MLT}(\alpha=0.5)$ or CGM, we are able to compute only "complete" $\operatorname{MLT}(\alpha=0.5)$ and "complete" FST models (the same treatment of convection in the interior structure and in the atmosphere). The internal structure of models labeled with MLT $\alpha=0.1,0.3,0.7$, and 1.75 has been computed until $\tau=1$ with these $\alpha$ values, and with boundary conditions for temperature and pressure $((T, P)(\tau=1))$ provided by the the $\operatorname{MLT}(\alpha=0.5)$ atmosphere model.

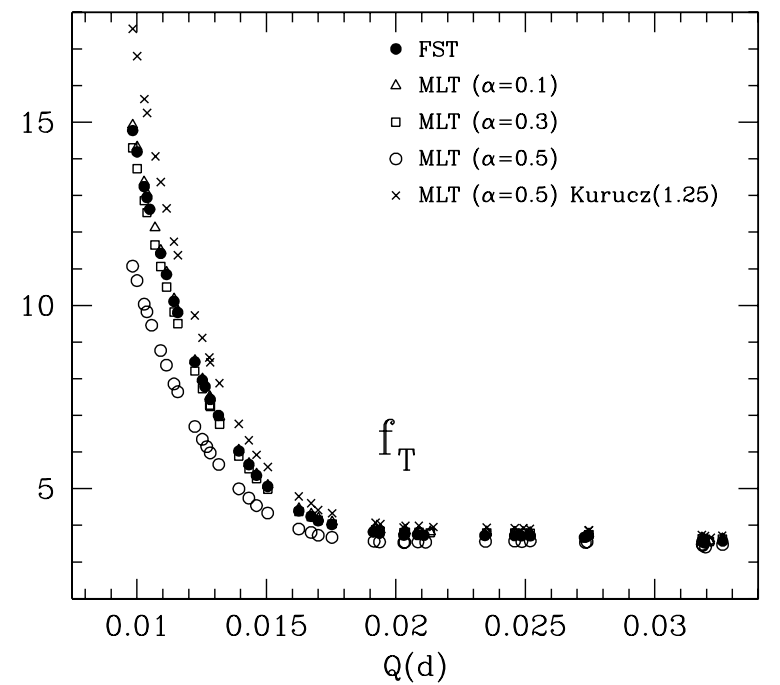

Fig. 3. The non-adiabatic quantity $f_{\mathrm{T}}=\left|\delta T_{\text {eff }} / T_{\text {eff }}\right|$ as a function of the pulsation constant $Q$ (in days) for modes with spherical degree $\ell=$ $0,1,2$, and 3 . These modes correspond to $1.8 M_{\odot}$ models in the same HRD location and computed with different convection treatment. The meaning of the labels is the same as in Fig. 1. The label MLT $(\alpha=0.5)$ Kurucz (1.25) refers to a model computed with $\operatorname{MLT}(\alpha=0.5)$ in the interior computation and Kurucz (1993)'s atmosphere models.

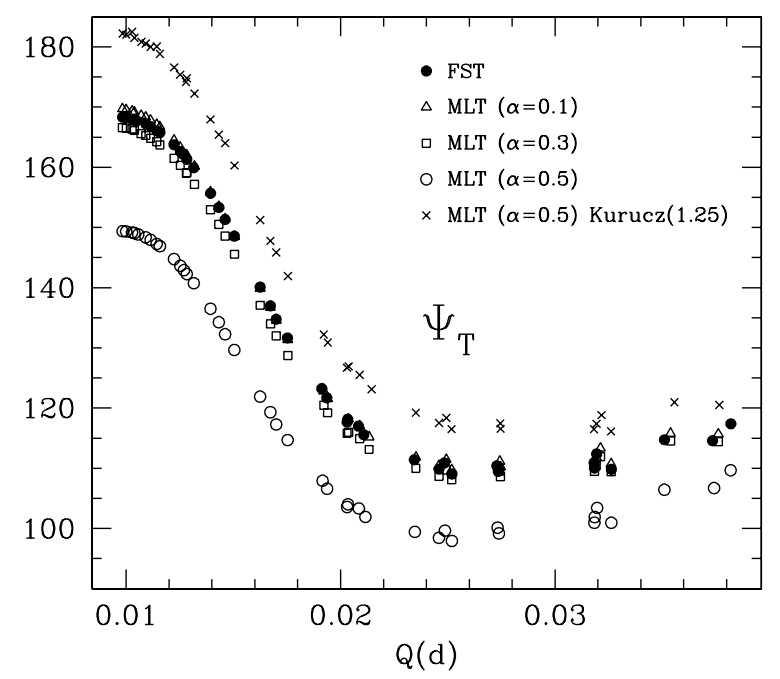

Fig. 4. As in Fig. 3 for the phase-lag (in degrees) between the local effective temperature variation and the radial displacement.

$\alpha$ increases, while the temperature gradient decreases. On the other hand, as pointed out by Canuto (1996), the energy fraction transported by convection in FST models is much smaller than that of MLT treatment in the case of low efficiency convection.

In next sections we analyze the consequences of these different structures on the non-adiabatic observables, and hence on the multi-color photometry as tool to mode identification.

\subsection{Non-adiabatic results}

We present the non-adiabatic results obtained for models with a given mass $\left(1.8 M_{\odot}\right)$ and effective temperature $\sim 7400 \mathrm{~K}$. In Figs. 3 and 4 , we plot the relative amplitudes $\left(f_{\mathrm{T}}\right)$ and phases $\left(\psi_{\mathrm{T}}\right)$ as a function of the pulsation constant $Q . Q=$ $P \sqrt{\left(R_{\odot} / R\right)^{3}\left(M / M_{\odot}\right)}$, with $P$ the period in days. In $\delta$ Scuti stars $Q \simeq 0.033$ days for the fundamental radial mode. The 
models used in Figs. 3 and 4 are the same as in Figs. 1 and 2 for the same kind of convection, and for all of them $\tau_{\text {dyn }} / \tau_{\text {dyn, } \odot}=$ $\sqrt{\left(R / R_{\odot}\right)^{3}\left(M_{\odot} / M\right)}=3.104282$.

The results presented in Figs. 3 and 4 confirm that the nonadiabatic quantities (particularly the phase-lag $\psi_{\mathrm{T}}$ ) are very sensitive to the convection treatment. As the value of the MLT- $\alpha$ parameter increases, the phase-lags decreases. Furthermore, for low values of the MLT- $\alpha$ parameter, the $\psi_{\mathrm{T}}$ value for high radialorder modes remain close to the adiabatic one $\left(180^{\circ}\right)$, as already discussed by Moya et al. (2004) and Dupret et al. (2005).

The $f_{\mathrm{T}}$ and $\psi_{\mathrm{T}}$ values for FST model are in between those obtained for $\operatorname{MLT}(\alpha=0.1)$ and $\operatorname{MLT}(\alpha=0.3)$ models. In all our models except one, we use NEMO atmosphere models with a low efficient convection (FST or MLT- $\alpha=0.5$ ). The exception is for the model with an interior structure computed with $\operatorname{MLT}(\alpha=0.5)$ up to $\tau=1$ and with the boundary conditions and the atmosphere structure given by $\alpha=1.25$ Kurucz's atmosphere models. The results provided by this last model and by the "complete" $\operatorname{MLT}(\alpha=0.5)$ model are significantly different. This shows the relevance of treating the interior and the atmosphere consistently.

As the effect of the temperature gradient on mode excitation is concerned, we state that for models with very inefficient convection, i.e. FST, as well as $\operatorname{MLT}(\alpha=0.1)$ and $\operatorname{MLT}(\alpha=0.3)$, only modes with $Q>0.014 \mathrm{~d}$ are excited, whereas for $\operatorname{MLT}(\alpha=0.5)$ this limit moves to higher frequencies, and modes with $Q>0.0125 \mathrm{~d}$ are excited as well.

In order to understand the behavior of $\psi_{\mathrm{T}}$ for different convection models, we plot the phase-lag between the total luminosity perturbation and the radial displacement $(\phi(\delta L)-\phi(\delta r))$ in Fig. 5 for the radial fundamental mode in the same models as in Figs. 3 and 4. In deep regions $(\log T>5)$, the pulsation is quasi-adiabatic and the phase-lag is $180^{\circ}$. A first phase lag, quasi-independent of the convection treatment, occurs at $\log T=$ 4.8-4.6, in the He II partial ionization zone, and a second one appears in the $\mathrm{H}$ partial ionization zone $(4.2 \geq \log T \geq 4$.). This second phase lag increases as the MLT $\alpha$ parameter increases, which leads to phase-lag values close to $0^{\circ}$ at the stellar surface.

As shown in Dupret et al. (2005), the phase-lags can be interpreted on the basis of the following integral expression:

$$
\begin{aligned}
\Delta \phi(\delta L) & \cong-\int \mathfrak{R}\left\{\frac{\delta s / c_{\mathrm{v}}}{\delta L / L}\right\} \frac{c_{\mathrm{v}} T \sigma}{L} \mathrm{~d} m \\
& =-\int \mathfrak{R}\left\{\frac{\delta s / c_{\mathrm{v}}}{\delta L / L}\right\} \frac{4 \pi r^{2} \rho c_{\mathrm{v}} T \sigma}{L \mathrm{~d} \ln T / \mathrm{d} r} \mathrm{~d} \ln T
\end{aligned}
$$

This equation makes the different contributions to the phaselag through the star evident. Thus, a significant contribution can be generated in a region where the thermal-relaxation time is close to or larger than the pulsation period, and/or in a region where the pulsation is highly non-adiabatic. This last situation is found in the convective zone coinciding with the $\mathrm{H}$ partial ionization zone (HCZ), where $\mathfrak{R}\left\{\delta s L /\left(c_{\mathrm{v}} \delta L\right)\right\}$ has a high value due to the large opacity bump and to the temperature gradient. The sensitivity of the phase-lag to the convection treatment can be made more obvious by noting that the eigenfunctions $\delta L / L$, when written as a function of $T$ instead of as a function of mass, are essentially the same for different convection treatments in the equilibrium models. The main reason is that the opacity is essentially a function of temperature and is not sensitive to $\alpha$ for a given interval or temperature (see also Moya et al. 2003; Dupret et al. 2005). As shown in Fig. 2, what changes significantly from

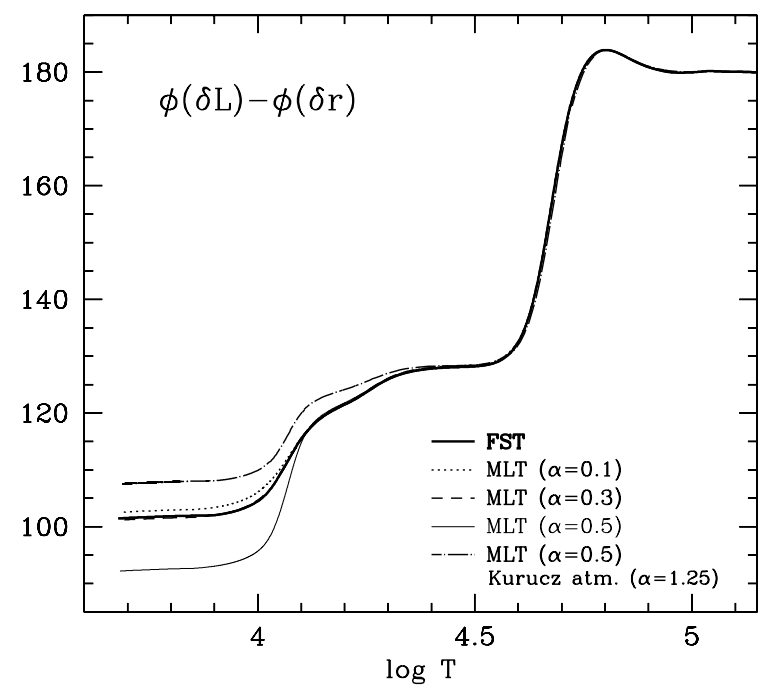

Fig. 5. Luminosity phase-lag $(\phi(\delta L / L)-\phi(\delta r / r)$ (in degrees) as a function of temperature inside the star for the radial $p_{1}$ mode corresponding to the same $1.8 M_{\odot}$ models as in previous figures.

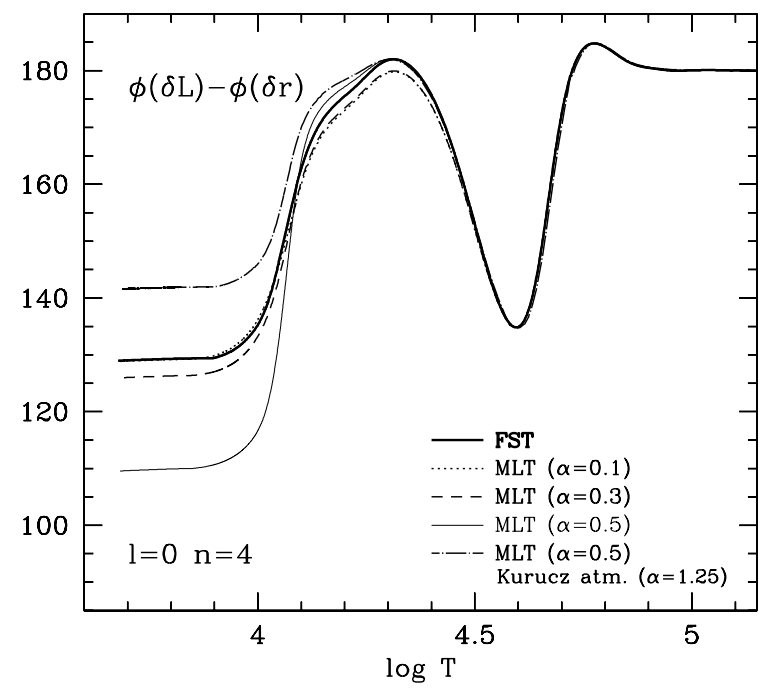

Fig. 6. As Fig. 5 for the $n=4$ overtone.

one model to another is the temperature gradient. When $\alpha$ increases, the temperature gradient in the HCZ decreases quickly. According to Eq. (5) (second line) the contribution to the phaselag from this zone increases with $\alpha$, and hence, the phase-lag can be considered as an indicator of the temperature gradient in the HCZ.

In Fig. 2, we see that the mean temperature gradient of the FST model is between the temperature gradients of the MLT models with $\alpha=0.1$ and $\alpha=0.3$; consequently, the phase lags predicted by FST models are between the $\alpha=0.1$ and $\alpha=0.3$ MLT ones (see Figs. 5 and 6).

It is well known that different modes probe the different layers of stellar interiors. Similarly we can expect, in principle, that the non-adiabatic phase-lags predicted for different modes probe the temperature gradient at different depths. It is evident from Fig. 2 that the exact shape of the temperature gradient predicted by FST models cannot be reproduced by MLT models, whatever the value of $\alpha$. Consequently, the phase lag predicted by FST models cannot be reproduced by the same MLT model in the whole frequency spectrum. This can be seen in Fig. 4. For periods close to that of the fundamental radial mode, the FST 


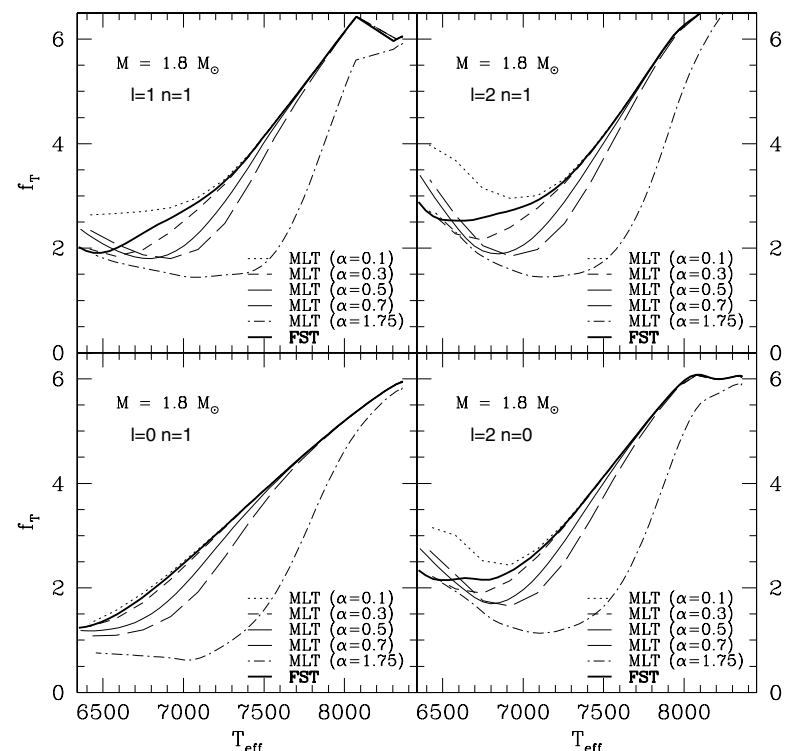

Fig. 7. The non-adiabatic quantity $f_{\mathrm{T}}=\left|\delta T_{\text {eff }} / T_{\text {eff }}\right|$ for four different modes $(\ell=0, n=1 ; \ell=1, n=1 ; \ell=2, n=0$, and $n=1)$ as a function of the effective temperature for the MS evolution of $1.8 M_{\odot}$ models computed with different convection treatments. The meaning of the labels is the same as in Fig. 1.

phase-lags are close to the $\operatorname{MLT}(\alpha=0.3)$ ones. But for smaller periods, the FST phase lags are closer to the $\alpha=0.1$ MLT ones. In Fig. 6 we plot $\phi(\delta L)-\phi(\delta r)$ of the $\mathrm{p}_{4}$ modes computed for the same models as in Fig. 5. As in the case of the $\mathrm{p}_{1}$ mode, we have $\mathrm{d} \phi(\delta L) / \mathrm{d} r<0$ in the partial ionization zones of $\mathrm{He}$ and $\mathrm{H}$, but, at variance with it, $\mathrm{d} \phi(\delta L) / \mathrm{d} r>0$ in the intermediate region between the $\mathrm{He}$ and $\mathrm{H}$ partial ionization zones, so that the phase-lag of the $\mathrm{p}_{4}$ mode is closer to $180^{\circ}$. These positive values of $\mathrm{d} \phi(\delta L) / \mathrm{d} r$ are associated with the damping of the modes in this region. Different shapes of the eigenfunctions, depending on the mode, imply a different sensitivity of the phase lag to the convection treatment. Nevertheless, the differences between the phase lags of the FST and the $\alpha=0.1, \alpha=0.3$ MLT models are small whatever the mode, and it would be too optimistic to conclude that the present precision of observations for the phase lags would make it possible to distinguish between FST and MLT models with the lowest $\alpha$ values (see discussion below).

Finally, in Figs. 7-10 we present the sensitivity of $f_{\mathrm{T}}$ and $\psi_{\mathrm{T}}$ to the convection treatment as a function of the effective temperature for the 1.8 and $2.0 M_{\odot}$ main-sequence models. Comparing the MLT and FST curves, we see that the FST results are always between the $\alpha=0.3$ and $\alpha=0.1$ MLT results, closer to $\alpha=0.1$ for hotter models and to $\alpha=0.3$ for cold models. It is also evident that $\psi_{\mathrm{T}}$ is much more sensitive than $f_{\mathrm{T}}$ to the convection treatment in the equilibrium model. Furthermore, the phase-lags decrease as effective temperature decreases, and tends to zero more rapidly as convection efficiency increases. The latter result agrees with those obtained by Moya et al. (2004) and by Dupret et al. (2005) in the frozen convection approximation. We notice that $\psi_{\mathrm{T}}$ differences among different convection treatments are larger for the cold models than for the hottest ones. These results can be understood as follows: when the effective temperature decreases, the size of the convective envelope increases quickly and the gradient of temperature decreases. This is similar to increasing $\alpha$ and, for the same reasons, the phase change between the bottom and top of the convection zone increases. A second effect of decreasing effective temperature is to increase

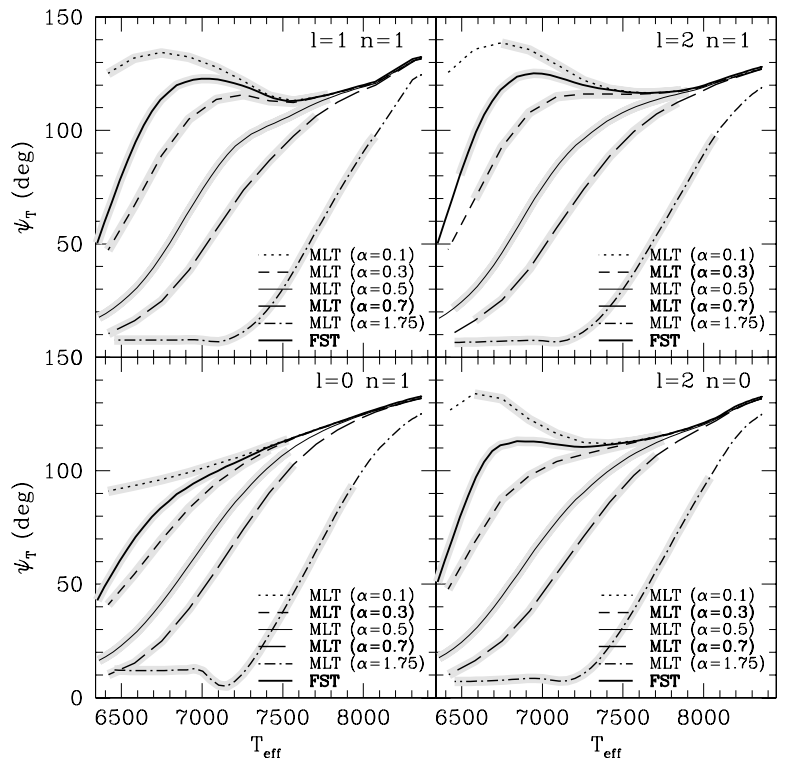

Fig. 8. Like Fig. 7 for the temperature phase-lag: $\psi_{\mathrm{T}}$. The gray bands indicate the models for which the corresponding $\ell, n$ mode is excited.

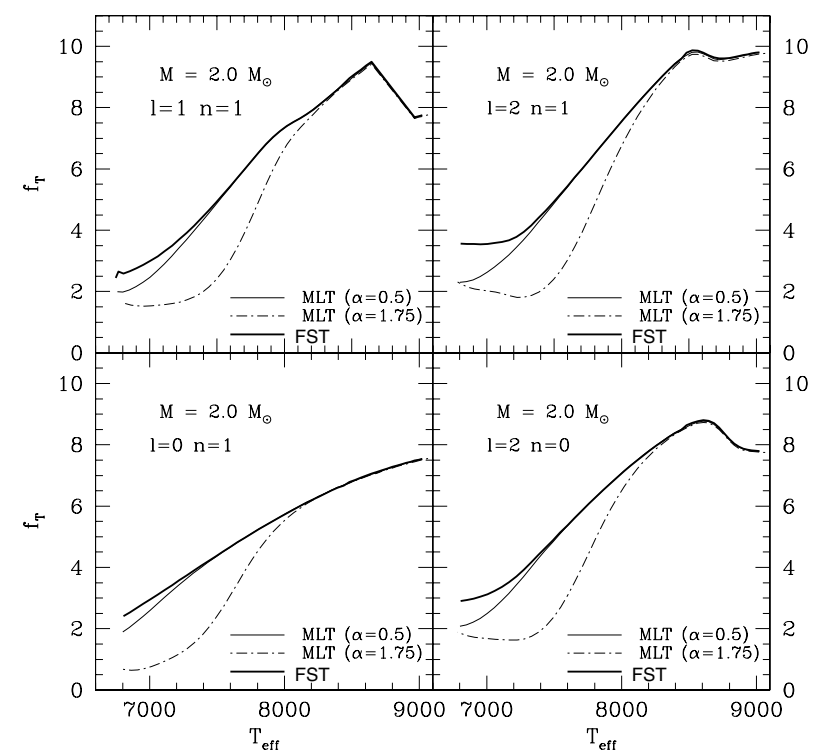

Fig. 9. Like Fig. 7 for $2.0 M_{\odot}$ models and for only three different convection treatments.

the opacity bump. Consequently, the associated bump of the nonadiabatic eigenfunctions is larger and also implies larger phase changes.

The value of $\psi_{\mathrm{T}}$ for FST models follows the $\operatorname{MLT}(\alpha=0.1)$ behavior until $T_{\text {eff }} \sim 6800 \mathrm{~K}$, then it suddenly changes its slope to follow the MLT $(\alpha=0.3) \psi_{\mathrm{T}}$ curve. This is explained by the properties of FST convection (see e.g. D'Antona et al. 2002), which induce a rapid change of the depth of the convective zone

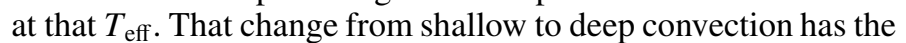
same effect on $\psi_{\mathrm{T}}$ as the increasing of $\alpha$ parameter in the MLT models. We note that FST $\psi_{\mathrm{T}}$ values for $T_{\text {eff }} \geq 6500 \mathrm{~K}$ models are between 90 and $140^{\circ}$, in good agreement with the typical observational values in $\delta$ Scuti stars and with the theoretical predictions of time-dependent convection models by Dupret et al. (2005).

For 2.0 $M_{\odot}$ models, where the convective zone is shallower than in $1.8 M_{\odot}$ ones, the effect of considering FST or 


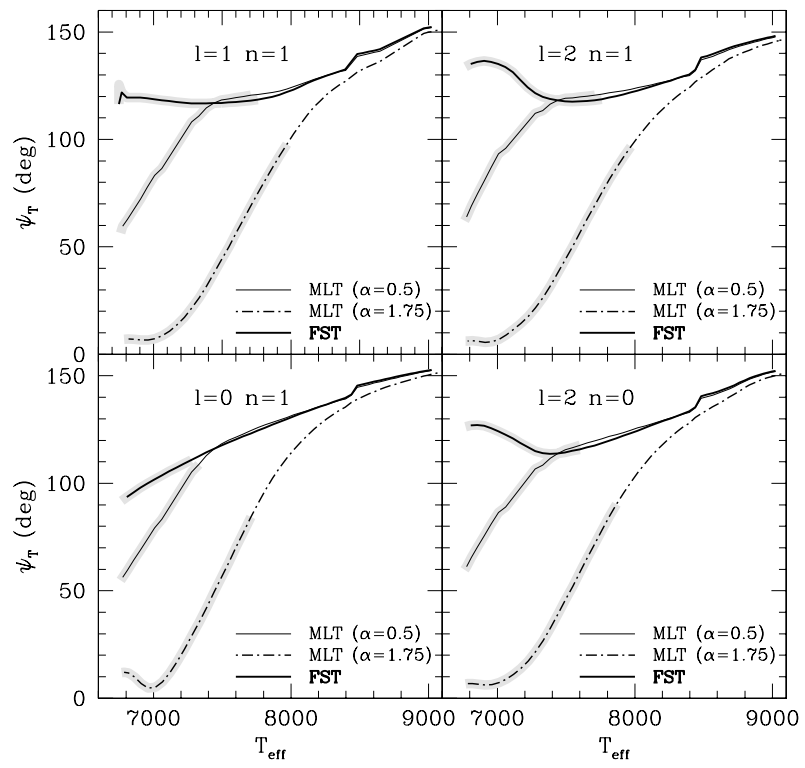

Fig. 10. As Fig. 9 for the temperature phase-lag: $\psi_{\mathrm{T}}$. The gray bands indicate the models for which the corresponding $\ell, n$ mode is excited.

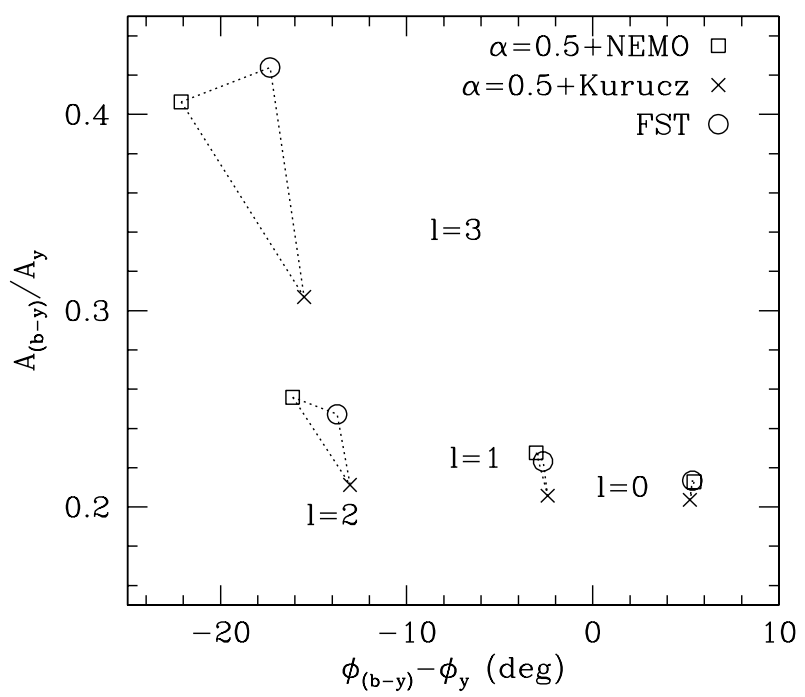

Fig. 11. Color-to-light amplitude ratio vs. the phase difference diagram for three $1.8 M_{\odot}$ models: complete FST model (circles), complete $\operatorname{MLT}(\alpha=0.5)$ model (squares), and MLT $(\alpha=0.5)$ model with Kurucz's (1993) atmospheres (crosses). Different groups correspond to different $\ell$ with pulsation constant $Q=0.033 \mathrm{~d}: \ell=3$, upper-left; $\ell=2$, lowerleft; $\ell=1$, lower-middle, and $\ell=0$, lower-right.

$\operatorname{MLT}(\alpha=0.5)$ is weaker than for $1.8 M_{\odot}$ models, for both the amplitude ratio and the phase lag. Nevertheless, FST models always show $\psi_{\mathrm{T}}>100^{\circ}$, while for $\operatorname{MLT}(\alpha=0.5) \psi_{\mathrm{T}}$ decreases down to $\sim 50^{\circ}$ for lower effective temperatures. High $\alpha$ values provide, as before, phase lags that are too low.

\subsection{Multi-color photometry}

The above-mentioned results directly affect the phase differences and amplitude ratios between different photometric passbands. More precisely, as introduced in Sect. 2.5, two different ingredients allow us to determine the magnitude variations in different passbands: the non-adiabatic normalized amplitudes and phases of effective temperature variation $\left(f_{\mathrm{T}}\right.$ and $\left.\psi_{\mathrm{T}}\right)$, and the dependence of the monochromatic flux and of limb darkening on the

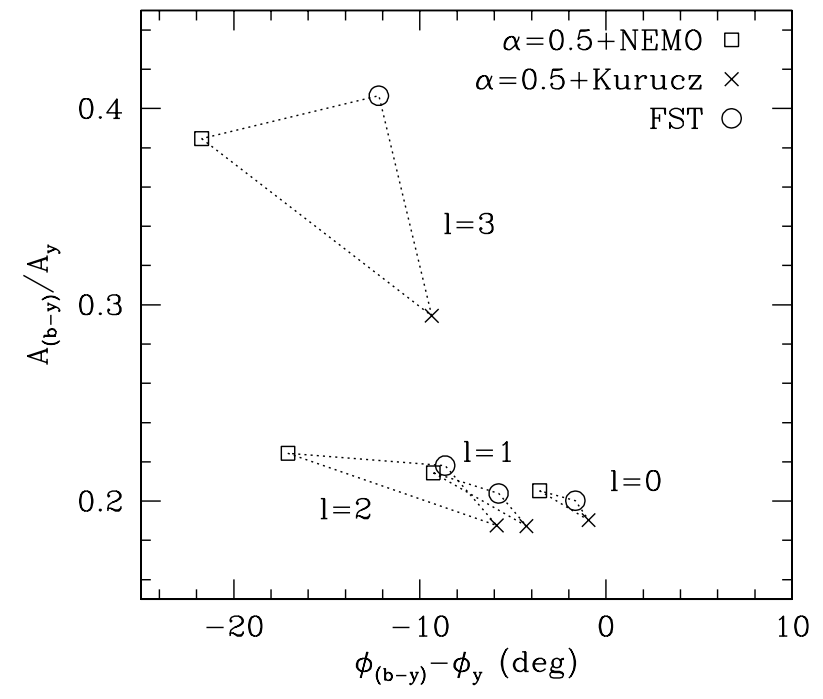

Fig. 12. Like Fig. 11 but for a mode with pulsation constant $Q=$ $0.015 \mathrm{~d}$.

effective temperature and gravity. All these quantities, as mentioned above, depend on the adopted treatment of convection.

We consider here three families of atmosphere models: Kurucz's (1993) models with $\operatorname{MLT}(\alpha=1.25)$ treatment and the two NEMO atmosphere models (Heiter et al. 2002) with $\operatorname{MLT}(\alpha=0.5)$ and FST.

We first recall that, because of the dependence of Eq. (1) on the degree $\ell$ of the mode, the comparison between theoretical and observed photometric amplitude ratios and phase differences makes $\ell$-identification possible. On the other hand, different convection treatments lead as well to different results, so that it is important to check how this affects mode identification. The classical diagrams for mode identification plot the amplitude ratio, $A_{(b-y)} / A_{y}$, versus $\phi_{(b-y)}-\phi_{y}$. Figure 11 shows the theoretical predictions for models with $T_{\text {eff }}=7400 \mathrm{~K}$, for modes with degrees $\ell=0$ to 3 and frequencies close to the fundamental radial mode. The results correspond to different models: one with $\operatorname{MLT}(\alpha=0.5)$ treatment in the interior and in the atmosphere, a second one with $\operatorname{MLT}(\alpha=0.5)$ in the interior but $\operatorname{MLT}(\alpha=1.25)$ in the atmosphere and a third one with FST treatment both in the interior and the atmosphere. We see that the regions corresponding to different $\ell$ are still clearly disjointed in this diagram when different convection treatments are considered. Moreover, we note that the typical precision of photometric methods, i.e. a few degrees for the phases and a few percent for the amplitude ratios, should allow different convection treatments to be differentiated. For the highest frequencies, however, this will only be possible for $\ell>2$ (Fig. 12).

A second important point is to examine the respective effects of the non-adiabatic calculations and of the atmosphere models on photometric amplitudes and phases. In Figs. 13 and 14 we plot the amplitude ratios and the phase differences as a function of the effective temperature for three model families: $i$ ) models with the FST treatment of convection in the interior and the atmosphere, and for which the monochromatic fluxes and limb darkening used in the transformation from theoretical to observational plane were computed with the same kind of model atmosphere as in the stellar modeling. ii) "Complete" $\operatorname{MLT}(\alpha=0.5)$ models, with a consistent transformation to the observational plane. iii) Hybrid modes, built by taking the non-adiabatic results $f_{\mathrm{T}}$ and $\psi_{\mathrm{T}}$ of "complete" $\operatorname{MLT}(\alpha=0.5)$ models, but with 


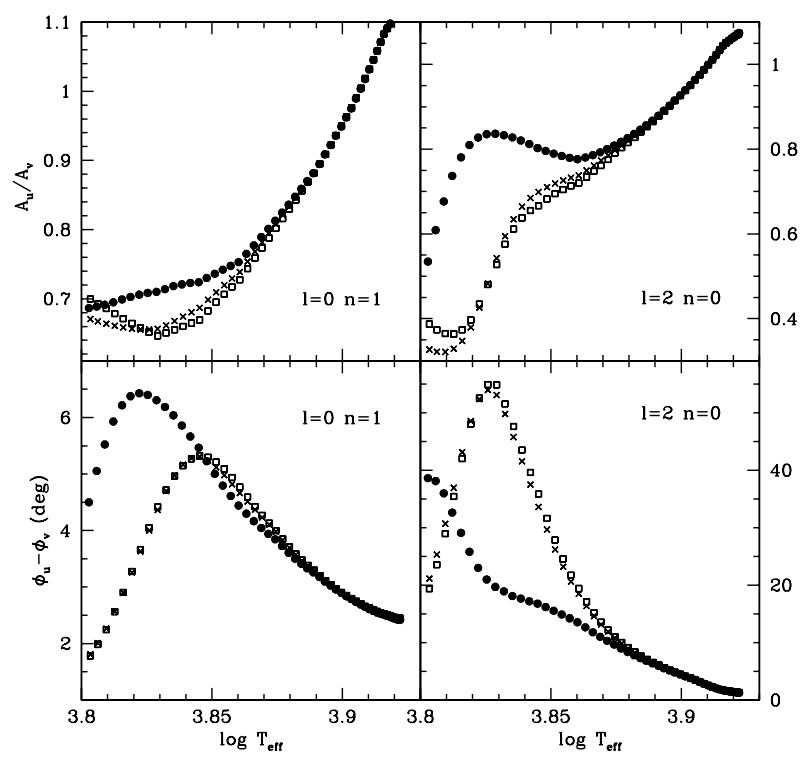

Fig. 13. Photometric amplitude ratio (upper panels) and phase lag (lower panels) for the $u$ and $v$ passbands for two different modes $(\ell=0$, $n=1$ and $\ell=2, n=0)$ vs. $T_{\text {eff }}$ for the MS evolution of $1.8 M_{\odot} \bmod -$ els. Solid circles correspond to complete FST models with the color transformation provided by FST atmosphere models. Open-squares refer to complete $\operatorname{MLT}(\alpha=0.5)$ with the color transformation given by $\operatorname{MLT}(\alpha=0.5)$ atmospheres, and crosses corresponds to complete $\operatorname{MLT}(\alpha=0.5)$ and the color transformation given by FST atmosphere models.

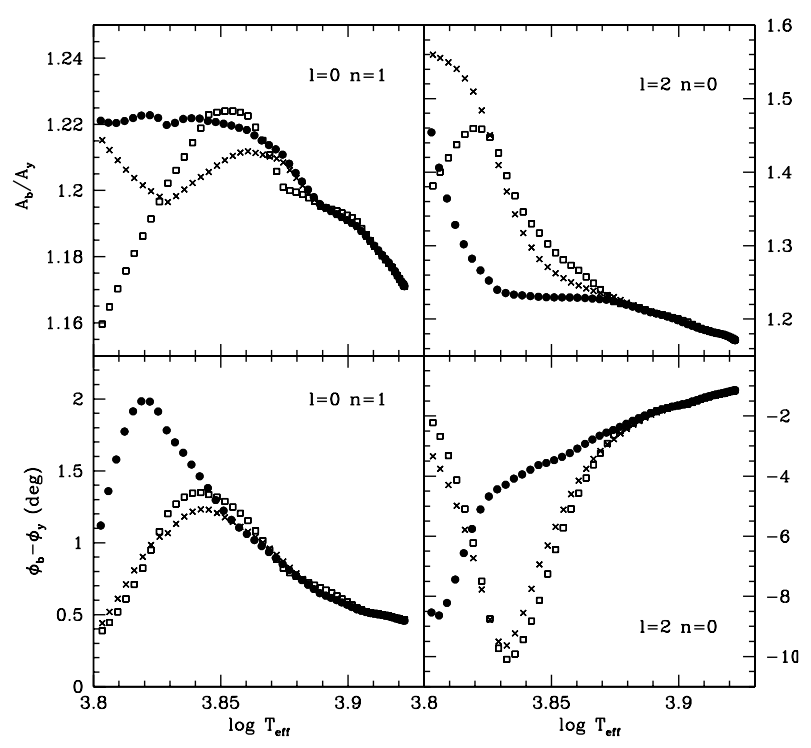

Fig. 14. Like Fig. 13, but for the $b$ and $y$ passbands.

the color transformations (Eq. (1)) obtained from the FST atmospheres.

Figure 13 shows the amplitude ratio and phase differences between $u$ and $v$ passbands, and Fig. 14 those between $b$ and $y$ passbands. Note that scales and ranges in those figures are not the same and that the effect of different convection treatments are much greater for the $u$ and $v$ passbands than for the $b$ and $y$ ones. We note another interesting result in both sets of figures: MLT and hybrid models show very close phase differences, and hence, the photometric phase differences mainly provide information about the non-adiabatic results. As shown in the previous section, $\psi_{\mathrm{T}}$ essentially depends on the temperature

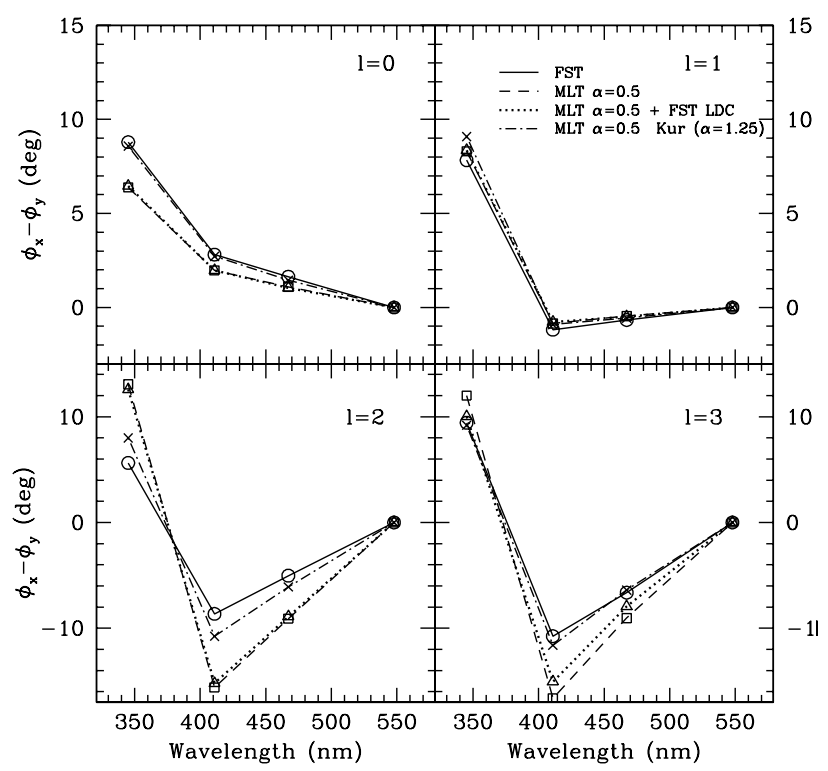

Fig. 15. Theoretical monochromatic phase differences $\phi_{u, v, b, y}-\phi_{y}$ as a function of wavelength for $1.8 M_{\odot}$ models with $T_{\text {eff }}=6800 \mathrm{~K}$ and $\log g=3.816$. Each panel corresponds to a different degree $\ell$ for frequencies close to the fundamental mode. Solid line: FST interior,and FST atmosphere structure and color transformation. Dashed line: $\operatorname{MLT}(\alpha=0.5)$ interior, and $\operatorname{MLT}(\alpha=0.5)$ atmosphere structure and color transformation. Dotted line: $\operatorname{MLT}(\alpha=0.5)$ interior, $\operatorname{MLT}(\alpha=0.5)$ atmosphere structure, and color transformation and limb-darkening coefficients derived from FST atmosphere models (labeled by: FST LDC). Dot-dashed line: $\operatorname{MLT}(\alpha=0.5)$ interior, Kurucz $\operatorname{MLT}(\alpha=1.25)$ atmosphere structure and color transformation.

gradient in the HCZ. Therefore, this gradient can be constrained by comparing the theoretical and observed multi-color photometric phase differences.

The differences between MLT and FST treatments are greatest at $T_{\text {eff }} \sim 6800 \mathrm{~K}$. We chose a $1.8 M_{\odot}$ star, with $\log T_{\text {eff }}=3.83$ and $\log g=3.81$, and we compared the effect of different external layers treatment on the monochromatic phase differences and amplitude ratios. In Fig. 15 we show the phase differences for the ubvy bands as a function of the wavelength (a separate panel for each degree $\ell=0,1,2$, and 3). Different curves correspond to "complete" FST and MLT $(\alpha=0.5)$ models, MLT with FST color transformations, and MLT $(\alpha=0.5)$ with Kurucz-MLT $(\alpha=1.25)$ atmospheres and the corresponding color transformations.

We confirm that the atmosphere models used in color transformation do not have a significant impact on the monochromatic phase difference (even if Kurucz-MLT $(\alpha=1.25)$ tables - not included in Fig. 15 - are used), although the effect increases with $\ell$. The same type of plots of Fig. 15 are displayed in Fig. 16 for the monochromatic amplitude ratio. As already shown in Figs. 3 and 4 , the differences in $f_{\mathrm{T}}$ and $\Psi_{\mathrm{T}}$ between the different treatments of convection decrease as the mode frequency decreases. That is the reason the ratios $A_{x} / A_{y}$ and phase differences $\phi_{x}-\phi_{y}$ for the modes with frequencies closest to the fundamental one presented in Figs. 16 and 15 are so similar for the different treatments of the external layers, and it is why the differences increase with the frequency (or degree $\ell$ ). We think that we must not overinterpret the results obtained for models mixing MLT $(\alpha=0.5)$ in the interior with Kurucz-MLT $(\alpha=1.25)$ atmospheres. First, because, as already mentioned in Sect. 3.1, by mixing very different convection treatments, we get unforeseeable results such as those shown in Fig. 4, where the high value of $\Psi_{\mathrm{T}}$ is probably due to a large contribution to the 


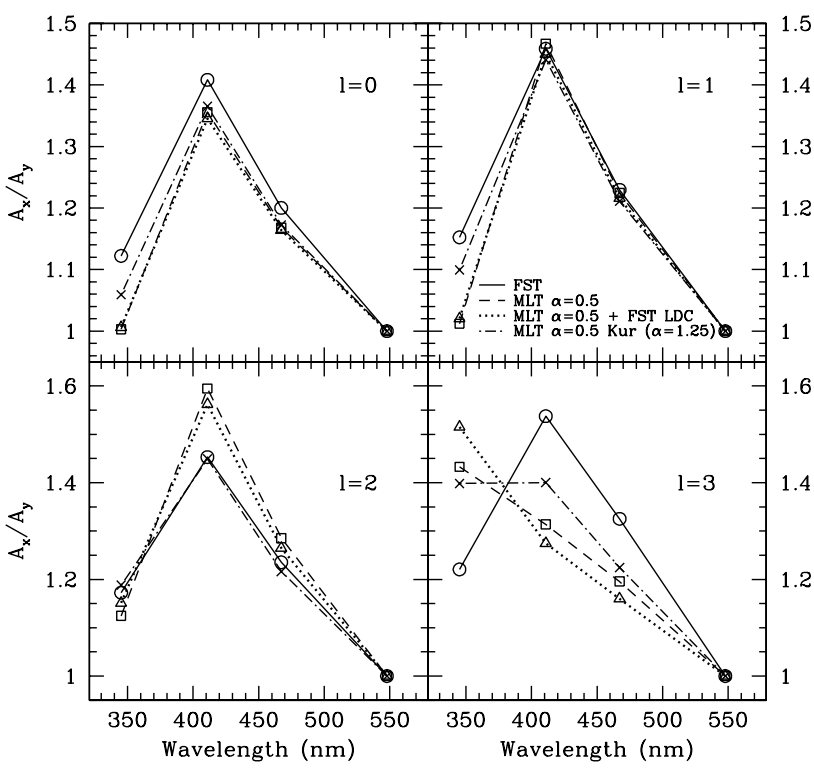

Fig. 16. Like Fig. 15 but for monochromatic amplitude ratio $A_{u, v, b, y} / A_{y}$.

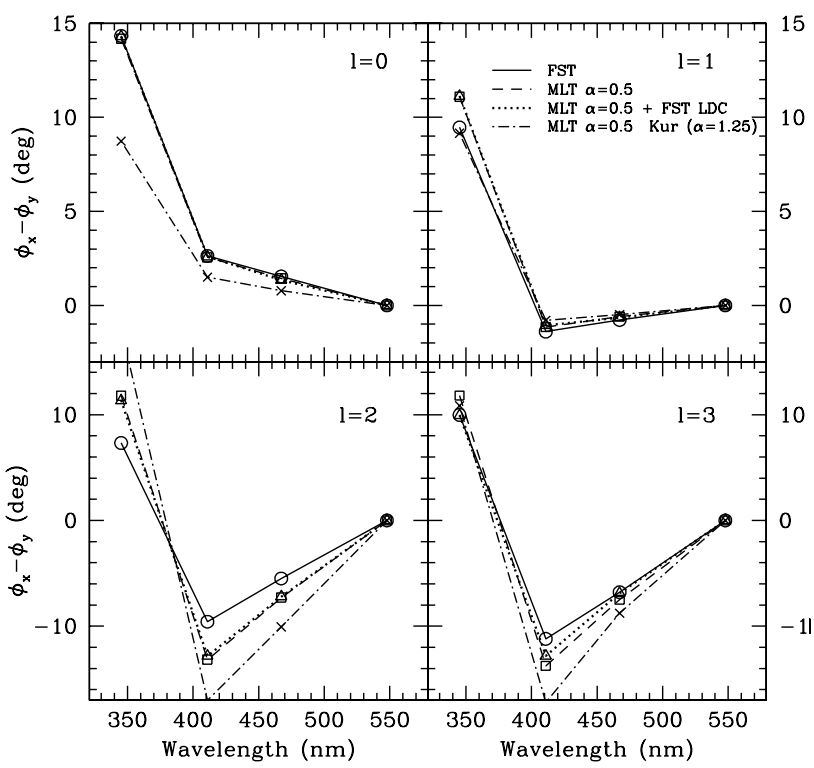

Fig. 17. Like Fig. 15 but for $1.8 M_{\odot}$ models with $T_{\text {eff }}=6750 \mathrm{~K}$ and $\log g=3.802$.

temperature gradient by changing from $\alpha=0.5$ to $\alpha=1.25$ if that happens in an over-adiabatic layer. Moreover, we must take into consideration that the Kurucz grid is sparser than the NEMO one, hence the derivatives with respect to $T_{\text {eff }}$ and $\log g$ involved in $\phi_{x}$ and $A_{x}$ are determined with greater uncertainty. These facts can be seen in Figs. 17 and 18, which are similar to Figs. 15 and 16 , but for a slightly different $1.8 M_{\odot}$ evolutionary state. These stellar models are only $50 \mathrm{~K}$ cooler than the previous ones, and their gravity is different by $\Delta \log g=0.014$. Now, the behavior of $A_{x} / A_{y}$ and $\phi_{x}-\phi_{y}$ for the MLT $(\alpha=0.5)+$ Kurucz$\operatorname{MLT}(\alpha=1.25)$ model has changed, and the differences with FST models have significantly increased.

Both the atmosphere models and non-adiabatic results can play a significant role in the amplitude ratios, mainly for higherdegree modes. Information on the flux and limb-darkening could thus be extracted from the amplitude ratios if the nonadiabatic predictions are known with a sufficiently high degree of confidence.

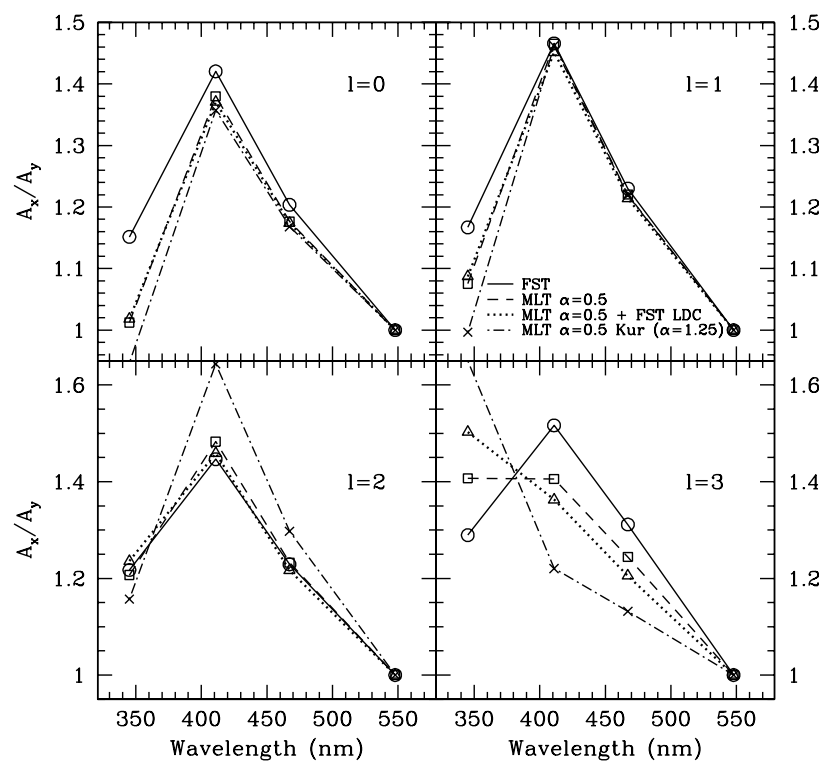

Fig. 18. Like Fig. 16 but for $1.8 M_{\odot}$ models with $T_{\text {eff }}=6750 \mathrm{~K}$ and $\log g=3.802$.

\section{Application to the $\delta$ Scuti star AN Lyn}

AN Lyn is a medium-amplitude $\delta$ Scuti star discovered by Yamasaki et al. (1981). Subsequent investigations have revealed that it is a multi-periodic $\delta$ Scuti (Rodriguez et al. 1997b) with a peculiar light curve (Rodriguez et al. 1997a). Rodriguez et al. (1997b) identified three independent frequencies: $v_{1}=$ $10.1756 \mathrm{c} / \mathrm{d}, v_{2}=18.1309 \mathrm{c} / \mathrm{d}$, and $v_{3}=9.5598 \mathrm{c} / \mathrm{d}$. Two of them $\left(v_{1}\right.$ and $v_{2}$ ) have been confirmed by Zhou (2002). Moreover, the amplitude of the dominant frequency $\left(v_{1}\right)$ seems to show variations on a timescale of years (Zhou 2002). Precise amplitudes and phases in Strömgren uvby photometry were derived by Rodriguez et al. (1997b), so we will consider these observational results to apply to our theoretical models.

The Strömgren indices, together with the calibration software TempLoGv2 (Stütz \& Nendwich 2002), provide the following global parameters: $T_{\mathrm{eff}}=7150 \mathrm{~K}, \log g=3.65$, and $[\mathrm{M} / \mathrm{H}]=-0.17$. By taking six different calibrations into account we obtain: $T_{\text {eff }}=7100 \pm 150 \mathrm{~K}, \log g=3.5 \pm 0.35$, and $[\mathrm{M} / \mathrm{H}]=-0.17 \pm 0.03$. Even if the metallicity resulting from this calibration is sub-solar, we do the computations with classical solar composition and NEMO atmospheres for $[\mathrm{M} / \mathrm{H}]=0$ and microturbulence velocity $\xi_{\mathrm{t}}=2 \mathrm{~km} \mathrm{~s}^{-1}$. There are several reasons for these choices: first of all, the value of metallicity provided by the photometric calibration is quite uncertain; second, Barban et al. (2003) computed the limb-darkening coefficients only for that metallicity and $\xi_{\mathrm{t}}$; and finally, the goal of this section is not to precisely fit the AN Lyn observations but to analyze how the choice of different treatments of the convective outer layers may affect the mode identification.

We computed a small grid of stellar models for $Z=0.02$, $X=0.7$ chemical composition, and for masses from 1.70 to $2.20 M_{\odot}$. The best fit of $T_{\text {eff }}(7166 \mathrm{~K}), \log g(3.76)$, main frequency and photometric features (amplitude ratios and phases) is obtained for $M=2.0 M_{\odot}$ at $\tau=1.10^{9} \mathrm{yr}, X_{\mathrm{c}}=0.145$, a mass of the convective core $M_{\mathrm{cc}} / M=0.18$, and a density contrast $\rho_{\mathrm{c}} / \bar{\rho} \sim 860$. As in previous papers concerning AN Lyn, we identify $v_{1}$ as an $n=2$ radial mode. Its second frequency, however, is affected by errors too large to be clearly identified. For this $2 M_{\odot}$ model, this frequency could correspond to a $\ell=3 n=2$ mode. 


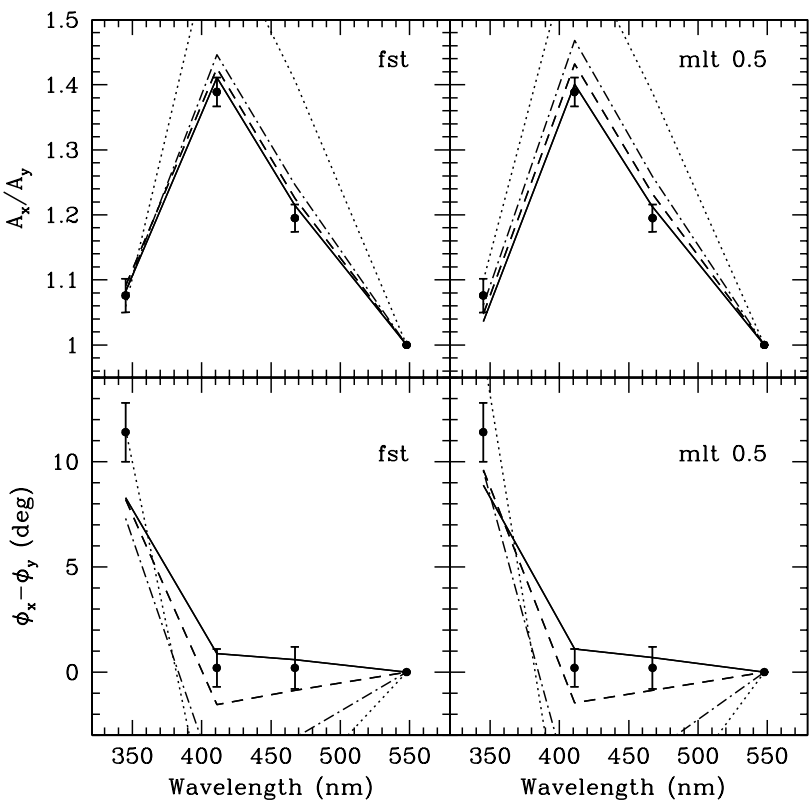

Fig. 19. Photometric amplitude ratios $A_{u, v, b, y} / A_{y}$ (lower panels), and photometric phase differences $\phi_{u, v, b, y}-\phi_{y}$ (upper panels) for different models of AN Lyn. Lines refer to theoretical predictions for $\ell=0$ (solid line), $\ell=1$ (dashed line), $\ell=2$ (dot-dashed line), and $\ell=3$ (dotted line), and the error bars represent the observations for $v_{1}$.

The four panels of Fig. 19 show the amplitude ratios and phases for the different (uvby) bands, which were computed for complete MLT and FST models and transformed to the observational plane by using the limb-darkening coefficients and integrals for the corresponding model atmosphere. We also checked the effect of combining a kind of atmosphere (MLT) in the stellar model with the color and limb-darkening transformations derived from FST model atmosphere. As already pointed out, the main effect is due to the temperature gradient in the external layers, and the effect of using FST or MLT based limb-darkening coefficients is negligible.

We also computed $\operatorname{MLT}(\alpha=1.25)$ models, consistently using the Kurucz model atmosphere grids and the corresponding limb-darkening coefficients. The temperature gradient derived from these models are unable to predict the expected amplitude ratios, especially in the $u$ band. Differences between FST or $\operatorname{MLT}(\alpha=0.5)$ are very small and not observationally disentangled. This result is not surprising, given the small differences in the theoretical non-adiabatic quantities shown in Figs. 9 and 10 for a $2 M_{\odot}$ star.

\section{Conclusions}

Mode-identification methods for $\delta$ Scuti pulsations are based on the non-adiabatic quantities $f_{\mathrm{T}}$ and $\psi_{\mathrm{T}}$, which are highly sensitive to the characteristics of the surface convective zone. The observed phase lag between light variation and the displacement originates in two different regions: the HeII partial ionization zone, where the $\kappa$ mechanism drives oscillations, and the HI and HeI partial ionization region, which coincides with a convective zone (HCZ). While the contribution from the HeII region mainly depends on its depth, and therefore on the evolutionary state, that of $\mathrm{HCZ}$ is very sensitive to the temperature gradient as well and, therefore, to the convection treatment. In this paper we have studied the role of convection in the external layers by comparing the low-efficiency convection models FST and
$\operatorname{MLT}(\alpha=0.5)$. The novelty of our analysis is that, for the first time, the model atmosphere used in the pulsation analysis was computed with the same treatment of convection as in the internal structure.

This study has shown that:

- Even if the FST and MLT $(\alpha=0.5)$ model atmospheres provide similar stellar spectra and limb darkening (Heiter et al. 2002; Barban et al. 2003), the non-adiabatic observables $f_{\mathrm{T}}$ and $\psi_{\mathrm{T}}$ clearly reflect the thermal structure of the inner layers of FST and MLT $(\alpha=0.5)$ over-adiabatic regions, showing differences of $\sim 20^{\circ}$ in the phase lag.

- Differences increase as $T_{\text {eff }}$ decreases, due to the different convective fluxes in FST and MLT treatments.

- For low efficiency of convection, the interaction pulsationconvection is negligible. If convection efficiency is low enough, we find phase lag values for the "frozen convection" approximation close to results obtained when taking the convection-pulsation interaction into account. Given that FST is less efficient than MLT, $\psi_{\mathrm{T}}$ for FST models remains higher than $100^{\circ}$ for lower $T_{\text {eff }}$ than in $\operatorname{MLT}(\alpha=0.5)$ ones.

- Differences between FST and $\operatorname{MLT}(\alpha=0.5)$ also decrease as stellar mass increases. With increasing stellar mass, the $\mathrm{HCZ}$ becomes shallower and shallower, so convection less and less efficient.

- The FST results cannot be reproduced with a single $\alpha$ value. Both $f_{\mathrm{T}}$ and $\psi_{\mathrm{T}}$ from FST are bracketed by $\operatorname{MLT}(\alpha=0.1)$ and $\operatorname{MLT}(\alpha=0.3)$, but the FST results are closer to $\operatorname{MLT}(\alpha=0.1)$ or to $\alpha=0.3$ ones depending on the frequencies. Furthermore, for higher $T_{\text {eff }}$ the FST behavior is closer to that of $\operatorname{MLT}(\alpha=0.1)$, but becomes closer to the $\operatorname{MLT}(\alpha=0.3)$ one as $T_{\text {eff }}$ decreases.

- The use of Kurucz model atmospheres with $\alpha=1.25$ in stellar models with $\alpha=0.5$ have shown that the differences due to the very external layers can introduce an uncertainty into the phase lag on the order of $40^{\circ}$ when compared with "complete" $\operatorname{MLT}(\alpha=0.5)$ models.

- As for $\psi_{\mathrm{T}}$, photometric phase differences in the Strömgren system are mainly sensitive to the temperature gradient in the HCZ, and are only slightly affected by the color transformation tables and limb-darkening functions provided by different model atmospheres. The amplitude ratios, in contrast, are affected by both the thermal structure and the color transformations. The $u$ and $v$ bands are in general the most affected: differences in $\phi_{u}-\phi_{y}$ on the order of 3-4 ${ }^{\circ}$ may be expected for $\ell=0$ or 2 , and differences of $10 \%$ to $20 \%$ for the amplitude ratio values.

- Differences between FST and $\operatorname{MLT}(\alpha=0.5)$ monochromatic phase differences and amplitude ratios are also frequencyand $\ell$-dependent. For frequencies close to the fundamental mode, the sensitivity to the convection treatment does not prevent identification of the mode degree $\ell$ from the classical amplitude ratio vs. phase differences diagram. But as frequency increases the mode identification $(\ell=$ 0,1 , or 2 ) becomes difficult due to the uncertainty on FST/MLT convection treatment. Adding radial velocity data could help, nevertheless, for distinguishing between FST and $\operatorname{MLT}(\alpha=0.5)$ approaches.

- The application of our study to the $\delta$ Scuti star AN Lyn provided results in good agreement with previous publications (Rodriguez et al. 1997a). Moreover, given the high mass derived for this star $\left(\sim 2.0 M_{\odot}\right)$ the effect of convection treatment is small, and it is not possible to distinguish 
between FST and MLT $(\alpha=0.5)$ on the base of the available observations.

In summary, the theoretical photometric amplitude ratios and phase differences between the photometric passbands are directly related to the non-adiabatic results (see Eq. (1)). Moreover, different modes probe different layers of the star. Hence, the temperature gradient in the superficial convection zone of intermediate-mass stars can be constrained by comparing these amplitude ratios and phase differences with observations. Such information cannot be obtained from the spectrum alone because spectral lines probe only regions of small optical depth.

Acknowledgements. We thank Dr. Rafael Garrido for kindly sending us his color-transformation program. J.M acknowledges financial support from the Prodex-ESA Contract Prodex 8 COROT (C90199).

\section{References}

Alexander, D. R., \& Ferguson, J. W. 1994, ApJ, 437, 879

Böhm-Vitense, E. 1958, Z. Astrophys., 46, 108

Balona, L. A., \& Stobie, R. S. 1979, MNRAS, 189, 649

Balona, L. A., \& Evers, E. A. 1999, MNRAS, 302, 349

Barban, C., Goupil, M. J., Van't Veer-Menneret, C., et al. 2003, A\&A, 405, 1095

Barklem, P. S., Stempels, H. C., Allende Prieto, C., et al. 2002, A\&A, 385, 951

Canuto, V. M. 1996, ApJ, 467, 385

Canuto, V. M., \& Mazzitelli, I. 1991a, ApJ, 370, 295

Canuto, V. M., \& Mazzitelli, I. 1991b, ApJ, 370, 295

Canuto, V. M., Goldman, I., \& Mazzitelli, I. 1996, ApJ, 473, 550

Castelli, F. 1996, in MASS, Model Atmospheres and Spectrum Synthesis, ed. S. J. Adelman, F. Kupka, \& W. W. Weiss, ASP Conf. Ser., 108, 85

Castelli, F., Gratton, R. G., \& Kurucz, R. L. 1997a, A\&A, 324, 432

Castelli, F., Gratton, R. G., \& Kurucz, R. L. 1997b, A\&A, 318, 841

Cox, J. P., \& Giuli, R. T. 1968, Principles of stellar structure (New York: Gordon and Breach)

Cugier, H., Dziembowski, W. A., \& Pamyatnykh, A. A. 1994, A\&A, 291, 143

D’Antona, F., Montalbán, J., Kupka, F., \& Heiter, U. 2002, ApJ, 564, L93

D'Antona, F., Cardini, D., Di Mauro, M. P., et al. 2005, MNRAS, 363, 847

Daszyńska-Daszkiewicz, J., Dziembowski, W. A., \& Pamyatnykh, A. A. 2003, A\&A, 407, 999
Daszyńska-Daszkiewicz, J., Dziembowski, W. A., Pamyatnykh, A. A., et al. 2005, A\&A, 438, 653

Dupret, M.-A., De Ridder, J., Neuforge, C., Aerts, C., \& Scuflaire, R. 2002, A\&A, 385, 563

Dupret, M.-A., De Ridder, J., De Cat, P., et al. 2003, A\&A, 398, 677

Dupret, M.-A., Grigahcène, A., Garrido, R., et al. 2005, MNRAS, 361, 476

Dziembowski, W. 1977, Acta Astron., 27, 203

Freytag, B., \& Steffen, M. 2004, in IAU Symp., ed. J. Zverko, J. Ziznovsky, S. J. Adelman, \& W. W. Weiss, 139

Garrido, R., Garcia-Lobo, E., \& Rodriguez, E. 1990, A\&A, 234, 262

Grevesse, N., \& Noels, A. 1993, in La formation des éléments chimiques, AVCP, ed. R. D. B. Hauck, \& S. Paltani

Heiter, U., Kupka, F., van't Veer-Menneret, C., et al. 2002, A\&A, 392, 619

Heynderickx, D., Waelkens, C., \& Smeyers, P. 1994, A\&AS, 105, 447

Iglesias, C. A., \& Rogers, F. J. 1996, ApJ, 464, 943

Itoh, N., \& Kohyama, Y. 1993, ApJ, 404, 268

Kupka, F., \& Montgomery, M. H. 2002, MNRAS, 330, L6

Kurucz, R. 1993, ATLAS9 Stellar Atmosphere Programs and $2 \mathrm{~km} \mathrm{~s}^{-1}$ grid, Kurucz CD-ROM No. 13, Cambridge, Mass.: Smithsonian Astrophysical Observatory, 13

Ludwig, H.-G., Freytag, B., \& Steffen, M. 1999, A\&A, 346, 111

Montalbán, J., D’Antona, F., Kupka, F., \& Heiter, U. 2004, A\&A, 416, 1081

Moya, A., Garrido, R., \& Dupret, M. A. 2003, in Asteroseismology Across the HR Diagram, ed. M. J. Thompson, M. S. Cunha, \& M. J. P. F. G. Monteiro, 549

Moya, A., Garrido, R., \& Dupret, M. A. 2004, A\&A, 414, 1081

Rodriguez, E., Gonzalez-Bedolla, S. F., Rolland, A., Costa, V., \& Lopez de Coca, P. 1997a, A\&A, 324, 959

Rodriguez, E., Gonzalez-Bedolla, S. F., Rolland, A., et al. 1997b, A\&A, 328, 235

Rogers, F. J., \& Nayfonov, A. 2002, ApJ, 576, 1064

Saumon, D., Chabrier, G., \& van Horn, H. M. 1995, ApJS, 99, 713

Smalley, B. 2004, in IAU Symp., ed. J. Zverko, J. Ziznovsky, S. J. Adelman, \& W. W. Weiss, 131

Smalley, B., Gardiner, R. B., Kupka, F., \& Bessell, M. S. 2002, A\&A, 395, 601 Stamford, P. A., \& Watson, R. D. 1981, Ap\&SS, 77, 131

Stütz, C., \& Nendwich, J. 2002, Vienna TempLogG v2 web interface: http://ams.astro.univie.ac.at/templogg/main.php

van't Veer-Menneret, C., \& Megessier, C. 1996, A\&A, 309, 879

van't Veer-Menneret, C., Bentolila, C., \& Katz, D. 1998, Contributions of the Astronomical Observatory Skalnate Pleso, 27, 223

Ventura, P., Zeppieri, A., Mazzitelli, I., \& D'Antona, F. 1998, A\&A, 334, 953

Watson, R. D. 1988, Ap\&SS, 140, 255

Yamasaki, A., Okazaki, A., \& Kitamura, M. 1981, PASP, 93, 77

Zhou, A.-Y. 2002, A\&A, 385, 503 